\title{
Dyspnoea on exertion in a child: A presentation of neuroendocrine hyperplasia of infancy
}

A C Jeevarathnum, MB BCh, FCPaed, Dip Allergy, MMed, Cert Paed Pulm, European Respiratory Diploma; A van Niekerk, MB BCh, MMed; D Parris, BSc, MB BCh, FCPaed, Dip Allergy; X Dearden, MB ChB, FCPaed, MMed, Dip Allergy; A Goga, PhD; R J Green, $\mathrm{PhD}$, DSc

Department of Paediatrics and Child Health, School of Medicine, Faculty of Health Sciences, and University of Pretoria and Steve Biko Academic Hospital, Pretoria, South Africa

Corresponding author: A C Jeevarathnum (acjeevarathnum@gmail.com)

Neuroendocrine hyperplasia of infancy (NEHI) syndrome is a rare type of interstitial lung disease in children, the exact incidence of which is unknown. The diagnosis of NEHI syndrome can be made on particular geographical findings on a computed tomography scan in a patient with a suggestive clinical picture, after exclusion of more common disorders. The gold standard of diagnosis is lung biopsy with bombesin staining. There is no current treatment for NEHI syndrome and symptoms usually resolve with time. We present a case of biopsy-proven NEHI in an infant who presented with dyspnoea on exertion.

S Afr Respir J 2017;23(2):39-41. DOI:7196/SARJ.2017.v23i2.159

Neuroendocrine hyperplasia of infancy (NEHI) is a type of childhood interstitial lung disease (chILD) first described in 2005. ${ }^{[1]}$ It is thought to be benign because it gradually resolves over time without treatment. Patients present with failure to thrive, tachypnoea, crackles and hypoxia. ${ }^{[1]}$ Infant pulmonary function testing is suggestive of air trapping. ${ }^{[2]}$ Lung-biopsy specimens indicate the presence of neuroendocrine cells in the terminal airways without other structural parenchymal disease. ${ }^{[3]}$ Since the publication by Brody et al. ${ }^{[4]}$ in 2010 the international literature has suggested that a diagnosis of NEHI syndrome can be made with confidence if the computed tomography (CT) scans have a particular geographical appearance in a patient who has a suggestive history and clinical findings. Patients with NEHI syndrome may incur significant morbidity with failure to thrive, and supplemental nutritional intervention is occasionally required. Symptoms are thought to gradually improve over time; however, more recently it has been shown that patients with NEHI syndrome may experience exacerbations of their illness, with significant air trapping. ${ }^{[5]}$

\section{Case report}

A 14-month-old male was referred for assessment of difficulty in breathing. The symptoms were predominantly dyspnoea on exertion, with shortness of breath, tachypnoea, and visible recessions noted by the parents from 6 months of age. There was no cough or wheeze associated with the dyspnoea on exertion. There was no significant family history of asthma or cystic fibrosis.

Clinically, he displayed no evidence of chronic lung disease, with no chest wall deformity, plethora, cyanosis or clubbing. He was hyperinflated with a barrel-shaped chest. No tachypnoea was noted at rest. He became tachypnoeic on exertion with a respiratory rate of $40 \mathrm{bpm}$. However, oxygen saturations were maintained post exercise. Severe sternal recessions were noted on exertion. There were no wheezes on auscultation at rest, or on exertion.
The patient's autoimmune screen and sweat tests were negative. His immune system displayed a partial mannan-binding lectin deficiency, with an absolute value of $201 \mathrm{ng} / \mathrm{mL}$. The abnormalities in his immune profile could not explain his symptoms.

His chest X-ray (CXR) showed marked hyperinflation with nonspecific reticular opacities in the right middle lobe and the lingua region; however, these features were not striking. His cardiac echocardiogram was normal. The HRCT chest scan (Fig. 1) showed ground glass opacities predominantly in the right middle lobe and lingula area, with no noted bronchiectasis. The bronchoalveolar

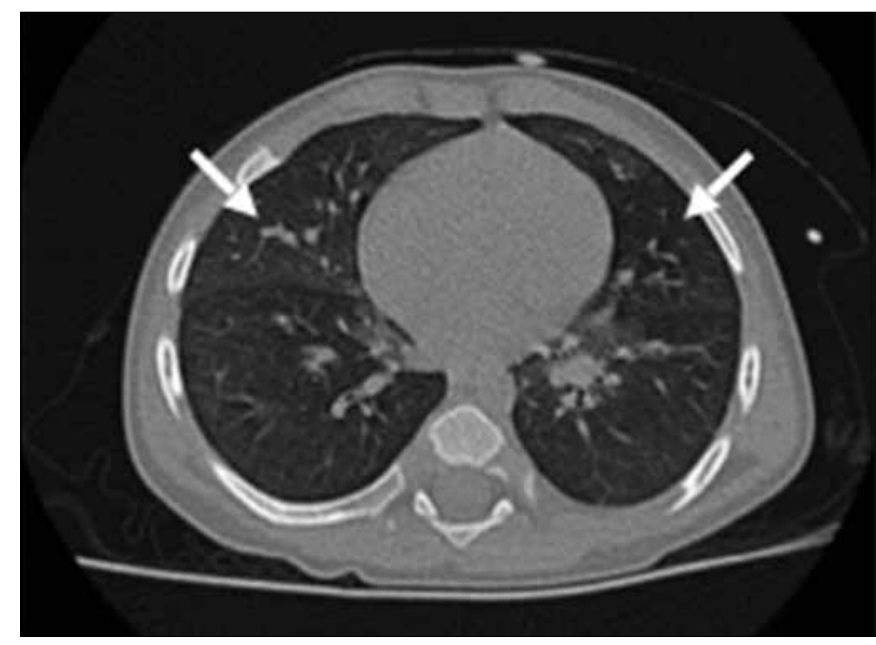

Fig. 1. Axial computed tomography chest scan depicting ground-glass opacities, predominantly in the right middle lobe, and lingula lesions (white arrows), which are characteristic of the geographical distribution associated with neuroendocrine hyperplasia of infancy (Courtesy of Prof. A van Niekerk, Department of Paediatrics and Child Health, Steve Biko Academic Hospital) 
lavage (BAL) ruled out an infective/inflammatory aetiology, but did not provide any additional diagnostic yield.

At this point, the most likely diagnosis was thought to be NEHI syndrome and the parents were counselled accordingly. A decision was then taken to proceed to a lung biopsy and undertake bombesin staining to confirm the diagnosis of NEHI after the parents sought confirmation of the diagnosis. The HRCT chest scan was in fact diagnostic and the lung biopsy was only undertaken because the parents sought histological confirmation. In retrospect, the diagnosis could have been made without lung biopsy confirmation. Fig. 2 depicts haematoxylin and eosin (H\&E) staining, which shows nonspecific interstitial changes. Bombesin staining was positive (Fig. 3). The clinical picture, CXR and CT chest scan findings, together with bombesin staining on lung biopsy specimens, were thought to be consistent with the diagnosis of NEHI.

The parents were counselled accordingly, and general measures including vaccinations, nutrition, and avoidance of environmental tobacco smoke were instituted. At the time of publication of this manuscript, the patient had not experienced any exacerbations relating to the NEHI syndrome

\section{Discussion}

NEHI syndrome forms part of the group of chILD diseases. The worldwide incidence of chILD in children is rare, estimated at 0.13 - 16.2 cases per $100000 .{ }^{[6]} \mathrm{NEHI}$ syndrome forms a subset of these rare disorders. The true incidence of NEHI syndrome is unknown; although it is assumed to be very rare, its true incidence could be underestimated. Retrospective lung biopsy data reveal an incidence of $10-14 \%$ of lung biopsy specimens in paediatrics, as per biopsy specimens of patients submitted for workup of chILD. ${ }^{[2,7]}$

The aetiology of NEHI syndrome is largely unknown. However, there is evidence of a heritable aspect to the syndrome. There are a number of familial cases of NEHI syndrome for which no genetic mutation has been found; ${ }^{[8]}$ however, Young et al. ${ }^{[9]}$ identified a previously unreported mutation in the NKX2.1 gene that may cause NEHI.

NEHI presents in otherwise healthy infants within the first few months to a year of life. ${ }^{[1,8,10]}$ The peak incidence is at 3.8 months of age. There is a slight male predominance. Patients present with tachypnoea with or without recessions, even at rest, and there is always a chronic history of tachypnoea that may precede viral infections. Cough and wheeze are not prominent features of NEHI. ${ }^{[1,10]}$ Certainly, tachypnoea seems to be the most common presenting feature, and one should be wary of NEHI syndrome in patients who present with tachypnoea as the only complaint.

On examination, patients are noted to be hyperinflated. There may be failure to thrive noted secondary to the increased work of breathing. Clubbing is not a feature and has not been reported in patients with NEHI. The CXR might be normal or show features of hyperinflation with perihilar opacities.

The approach to diagnosis of a patient with suspected NEHI syndrome would follow a similar algorithm, as set out by the American Thoracic Society for the workup of a patient with suspected interstitial lung disease. ${ }^{[11]}$ Firstly, more common causes, such as acute and chronic infections, asthma, cystic fibrosis, and primary immune deficiencies need to be ruled out. It is mandatory that these patients have a formal cardiac evaluation to rule out congenital cardiac causes.

If the initial workup is non-contributory, a CT chest scan should be undertaken. This is by far one of the most important investigations in revealing the diagnosis. It is well known that the diagnosis of NEHI syndrome can be made without a lung biopsy in those patients with a suggestive clinical syndrome. The characteristic HRCT findings of a patient with NEHI syndrome include ground-glass opacities in the right middle lobe, lingula, as well as the medial aspects of the upper and lower lobes; this occurs in the absence of other parenchymal abnormalities. ${ }^{[4,8]}$ These findings reduce the need for performance of lung biopsies in all patients who present with a suggestive syndrome.

In NEHI syndrome patients, pulmonary function testing (PFT) shows elevated functional residual capacity ${ }^{[2]}$ and, although PFT may play a role in suggesting the diagnosis, it cannot be used as a diagnostic method.

BAL specimens in patients with NEHI are non-inflammatory. A case series pilot study of 41 patients in 2013 including patients with cystic fibrosis, NEHI, follicular bronchiolitis, and controls revealed patients with NEHI had the lowest BAL white-cell count, a higher percentage of alveolar macrophages, and lower levels of interleukin $1-\beta$, macrophage inflammatory protein $-1 \beta$, and interleukin- 8 on BAL

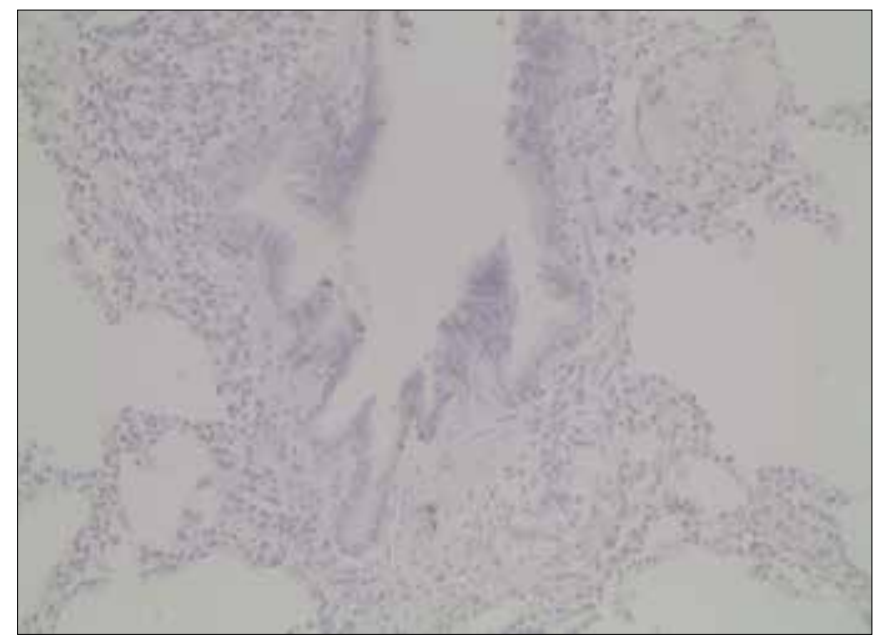

Fig. 2. HぬE lung biopsy staining showing non-specific inflammatory changes. (Courtesy of Dr Preea Pillay, Department of Anatomical Pathology, Ampath Laboratories)

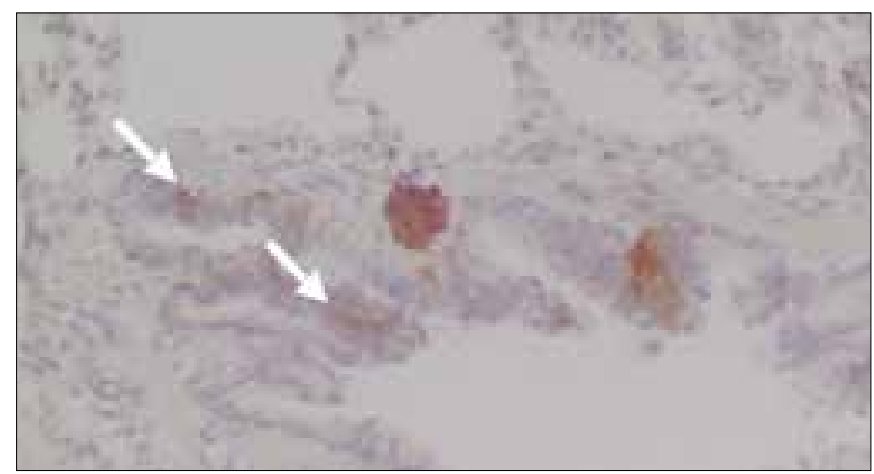

Fig. 3. Bombesin staining of lung biopsy, showing significant positive neuroendocrine cells (arrow heads). (Courtesy of Dr Preea Pillay, Department of Anatomical Pathology, Ampath Laboratories) 
compared with other patients in the study. With further research and validation of data in this series, BAL specimens may well be considered diagnostic in time to come. ${ }^{[12]}$

In some cases, where the typical features are not seen on a HRCT chest scan, a lung biopsy may be required to diagnose NEHI. The initial H\&E stains may show mild inflammatory changes. The chromogranin A staining used in some pathology laboratories does not sufficiently stain neuroendocrine cells in NEHI and therefore should be avoided. The hallmark of the lung-biopsy changes lies in bombesin staining and demonstration of pulmonary neuroendocrine cells..$^{[2]}$

There is no specific treatment of NEHI, and therefore treatment of patients with NEHI requires supportive measures including vaccinations, avoidance of environmental tobacco smoke, and provision of adequate nutrition. Steroids have shown little benefit in the management of patients. ${ }^{[1]}$

Most patients with NEHI demonstrate gradual improvement over time without specific treatment of the disease. However, recently it has been shown that patients with NEHI also display exacerbations of the disease, with increased air trapping being a predominant feature. Unfortunately, there is no conclusion on how to actively manage a patient with NEHI during an exacerbation. ${ }^{[5]}$ In the case series which described these exacerbations, oxygen was used in one case, and Zithromax with prednisone in the other; both showed resolution over time. ${ }^{[12]}$ As this syndrome gains more recognition in the international literature, management strategies during an exacerbation will probably be alluded to.

Acknowledgements: None.

Author contributions: ACJ wrote the manuscript. AVN reviewed the patient with ACJ. DP, XD, AG and RJG co-authored the manuscript.

Funding: None

Conflicts of interest: None
1. Deterding RR, Pye C, Fan LL, Langston C. Persistent tachypnea of infancy is associated with neuroendocrine cell hyperplasia. Pediatr Pulmonol 2005;40(2):157-165. http:// doi.org/10.1002/ppul.20243

2. Kerby GS, Wagner BD, Popler J, et al. Abnormal infant pulmonary function in young children with neuroendocrine cell hyperplasia of infancy. Pediatr Pulmonol 2013;48(10):1008-1015. http://doi.org/10.3410/f.718045206.793480909

3. Young LR, Brody AS, Inge TH, et al. Neuroendocrine cell distribution and frequency distinguish neuroendocrine cell hyperplasia of infancy from other pulmonary disorders. Chest 2011;139(5):1060-1071. http://doi.org/10.1378/chest.10-1304

4. Brody AS, Guillerman RP, Hay TC, et al. Neuroendocrine cell hyperplasia of infancy: Diagnosis with high-resolution CT. Am J Roentgenol 2010;194(1):238-244. http:// doi.org/10.2214/AJR.09.2743

5. Houin PR, Deterding RR, Young LR. Exacerbations in neuroendocrine cell hyperplasia of infancy are characterized by increased air trapping. Pediatr Pulmonol 2016(51):E9E12. http://doi.org/10.1002/ppul.23347

6. Hime NJ, Zurynski Y, Fitzgerald D, et al. Childhood interstitial lung disease: A systematic review. Pediatr Pulmonol 2015;50(12):1383-1392. http://doi.org/10.1002/ ppul.23183

7. Deutsch GH, Young LR, Deterding RR, et al. Diffuse lung disease in young children: Application of a novel classification scheme. Am J Respir Crit Care Med 2007;176(11):1120-1128. http://doi.org/10.1164/rccm.200703-393OC

8. Vece TJ, Young LR. Update of diffuse lung disease in children. Chest 2016;149(3):836845. http://doi.org/10.1378/chest.15-1986

9. Young LR, Deutsch GH, Bokulic RE, Brody AS, Nogee LM. A mutation in TTF1/ NKX2.1 is associated with familial neuroendocrine cell hyperplasia of infancy. Chest 2013;144(4):1199-1206. http://doi.org/10.1378/chest.13-0811

10. O'Connor MG, Wurth M, Young LR. Rare becomes more common: Recognizing neuroendocrine cell hyperplasia of infancy in everyday pulmonary consultations. Ann Am Thorac Soc 2015;12(11):1730-1732. http://doi.org/10.1513/AnnalsATS.201507$422 \mathrm{LE}$

11. Kurland G, Deterding RR, Hagood JS, et al. An official American Thoracic Society clinical practice guideline: Classification, evaluation, and management of childhood interstitial lung disease in infancy. Am J Respir Crit Care Med 2013;188(3):376-394. http://doi.org/10.1164/rccm.201305-0923ST.

12. Popler J, Wagner BD, Tarro HL, Accurso FJ, Deterding RR. Bronchoalveolar lavage fluid cytokine profiles in neuroendocrine cell hyperplasia of infancy and follicular bronchiolitis. Orphanet J Rare Dis 2013;8(1):175-184. http://doi.org/10.1186/17501172-8-175 